\title{
Color plasma oscillation in strangelets
}

\author{
Kei Iida \\ Department of Physics, University of Tokyo, 7-3-1 Hongo, Bunkyo, Tokyo 113-0033, Japan
}

(September 18, 2018)

\begin{abstract}
The dispersion relation and damping rate of longitudinal color plasmons in finite strange quark matter (strangelets) are evaluated in the limits of weak coupling, low temperature, and long wavelength. The property of the QCD vacuum surrounding a strangelet makes the frequency of the plasmons nearly the same as the color plasma frequency of bulk matter. The plasmons are damped by their coupling with individual excitations of particle-hole pairs of quarks, of which the energy levels are discretized by the boundary. For strangelets of macroscopic size, the lifetime of the plasmons is found to be proportional to the size, as in the case of the usual plasma oscillations in metal nanoparticles.
\end{abstract}

PACS numbers: 12.38.Mh, 25.75.-q

\section{INTRODUCTION}

At extremely high temperature and/or baryon density, quark-gluon plasmas [1] are considered to be energetically favorable as compared with hadronic matter. Such extreme conditions prevent us from confirming the presence of quark-gluon plasmas, although it is expected in interiors of neutron stars and in ultrarelativistic heavy-ion collisions. The intriguing possibility that strange quark matter, composed of $u, d$, and $s$ quarks, might be the ground state of the strong interaction was pointed out by Witten [2]. If this is correct, finite strange quark matter, which is usually referred to as strangelets, would be a more stable self-bound system than a ${ }^{56} \mathrm{Fe}$ nucleus. Then, disruption of neutron stars and highly energetic heavy-ion collisions could leave behind many strangelets. This might allow us to detect quark-gluon plasmas in the form of strangelets under various experimental situations [3 5].

The ground-state properties of strangelets were described by Farhi and Jaffe [6] within the MIT bag model; finitesize effects such as surface, Coulomb, and shell effects were taken into account. In ultrarelativistic heavy-ion collisions, however, the possibly formed strangelets should be accompanied by thermally excited states. Among various excitations, we take note of spin- and flavor-symmetric, longitudinal color plasmons [7,8] that represent collective counteroscillations of different color degrees of freedom. In a strangelet, these plasmons are expected not only to exhibit a dispersion relation dependent on the color dielectric property of the medium, but also to undergo a size-dependent damping as in the case of an optically excited metal cluster or nanoparticle. When the nanoparticle is small enough for the electrons to be nearly collisionless and for the electric dipole radiation to be of little significance but is too large for the shell structure to take effect, the surface plasmon excitations are damped in a time proportional to the size [9].10]. Their energy is resonantly absorbed by individual excitations of particle-hole pairs of the electrons having discrete energy levels due to the presence of the boundary. Notice that the quark-gluon plasma in a strangelet is fully relativistic in contrast to the electron gas in a nanoparticle. Consequently, new features should arise from spin-orbit interactions and from excitations involving antiquarks, both of which may affect the lifetimes of the color plasmons.

Longitudinal color plasma oscillations in relativistic and degenerate bulk matter of $u, d$, and $s$ quarks were investigated [7,8] by calculating the current-current correlation function within the random-phase approximation (RPA) which is valid in the weak-coupling limit. The dispersion law and damping for these oscillations are essentially the same as those examined by Jancovici [11] for electromagnetic plasma oscillations in a relativistic and degenerate electron gas. In either case, the mode properties are characterized by the corresponding plasma frequency and Landau damping as in a nonrelativistic plasma. In this paper, we estimate the long-wavelength, low-temperature properties of longitudinal color oscillation modes in a strangelet of macroscopic size, surrounded by the QCD vacuum, by incorporating the discrete energy levels of quarks into the current-current correlation function within the RPA. The vanishing color dielectric constant of the vacuum leads to the absence of the surface color charge; if present, it would modify the effective color electric field inside the strangelet according to a Mie-type formula. We thus find that the frequency of the longitudinal color plasmons in the strangelet is almost the same as that in bulk quark matter. The lifetime of the plasmons is determined by the dipole transitions from a quark state in the Fermi sea to an unoccupied one. It is shown that the quark particle-hole pairs involved damp the plasmons at a rate inversely proportional to the strangelet size. Beyond the RPA, however, the plasmons are further damped by collisions between quarks.

In Sec. II, the longitudinal polarization function is calculated up to one-loop order, allowing for the discrete quark 
states inside a strangelet. The resulting dispersion relation and damping rate of the longitudinal color plasma oscillation are described in Secs. III and IV, respectively. Concluding remarks are given in Sec. V.

\section{POLARIZATION}

We consider a spherical strangelet of radius $R$ and baryon number $A$, composed of a nearly noninteracting and uniform gas of $u, d$, and $s$ quarks and embedded in the QCD vacuum. The chemical potential $\mu_{i}$ of quarks of flavor $i$, including the rest mass $m_{i}$, is assumed to be independent of $i$. Such independence ensures flavor equilibrium among the quarks, since normally leptons have a vanishingly small chemical potential in the strangelet [6]. We work in units in which $\hbar=c=k_{B}=1$. We set $\mu_{i} \equiv \mu=300 \mathrm{MeV}, m_{u}=m_{d}=0$, and $m_{s}=0-300 \mathrm{MeV}$. The temperature $T$ of the system is taken to be in the range $0 \leq T \ll \mu$. The zero-temperature model for quark matter will thus be adopted below.

Let us now examine the properties of longitudinal color oscillation modes in a strangelet by calculating the corresponding proper polarization $\Pi_{00}$ in the RPA (one-loop order). At $T=0, \Pi_{00}$ can be expressed in terms of the correlation function of the time component of the quark current $\mathcal{J}_{\alpha}^{0}(\alpha=1, \ldots, 8)$ as

$$
\Pi_{00}(q)=-\frac{i}{V} \int_{V} d \mathbf{x} \int_{V} d \mathbf{y} \int d\left(x^{0}-y^{0}\right) e^{i q(x-y)}\left(\frac{g}{2}\right)^{2}\left\langle\left[\mathcal{J}_{\alpha}^{0}(x), \mathcal{J}_{\alpha}^{0}(y)\right]\right\rangle \theta\left(x^{0}-y^{0}\right)
$$

with

$$
\mathcal{J}_{\alpha}^{0}(x)=\lambda_{a b}^{\alpha} \bar{\psi}_{a i}(x) \gamma^{0} \psi_{b i}(x)
$$

where $q=(\omega, \mathbf{q})$ is the four-momentum transfer, $V=4 \pi R^{3} / 3$ is the volume of the strangelet, $g$ is the renormalized color coupling constant, $\psi_{a i}$ is the quark field of color $a$ and flavor $i, \lambda_{a b}^{\alpha}$ are the $\mathrm{SU}(3)$ generators, and $\langle\cdots\rangle$ denotes the expectation value in the ground state. The right-hand side of Eq. (1) is not summed over $\alpha$ and is invariant under change in $\alpha$. In the limit $V \rightarrow \infty$, this form of $\Pi_{00}(q)$ reduces to the usual form written for bulk uniform matter. Hereafter, we assume that the momentum transfer $|\mathbf{q}|$ is far smaller than $R^{-1}$.

The essential part of the present study is to incorporate the discrete quark levels into the one-loop order calculations of $\Pi_{00}$. For such calculations, we first write the quark field in the form of a mode expansion

$$
\psi_{a i}\left(x^{0}, \mathbf{x}\right)=\sum_{\xi \kappa}\left[c_{\xi \kappa a i} \Psi_{\xi \kappa a i}^{(+)}(\mathbf{x}) e^{-i \omega_{k i} x^{0}}+d_{\xi \kappa a i}^{\dagger} \Psi_{\xi \kappa a i}^{(-)}(\mathbf{x}) e^{i \omega_{k i} x^{0}}\right],
$$

where $\Psi_{\xi \kappa a i}^{(+)}$and $c_{\xi \kappa a i}$ are the spinor and the annihilation operator for the quark eigenstate of energy $\omega_{k i}=\sqrt{k^{2}+m_{i}^{2}}$ characterized by a set of the quantum numbers $\xi=\{k, j, m\}$, with wave number $k$, angular momentum $j$, and its $z$ component $m$, and by the integer $\kappa= \pm(j+1 / 2)$ corresponding to the parity $(-1)^{j \pm 1 / 2} ; \Psi_{\xi \kappa a i}^{(-)}$and $d_{\xi \kappa a i}$ are for the antiquark eigenstate of energy $\omega_{k i}$. The spinors $\Psi_{\xi \kappa a i}^{( \pm)}$can be obtained from the Dirac equation

$$
\left(i \gamma \cdot \nabla \pm \gamma^{0} \omega_{k i}-m_{i}\right) \Psi_{\xi \kappa a i}^{( \pm)}=0 \quad \text { for } r<R
$$

and the boundary condition

$$
-i \boldsymbol{\gamma} \cdot \hat{\mathbf{r}} \Psi_{\xi \kappa a i}^{( \pm)}=\Psi_{\xi \kappa a i}^{( \pm)} \quad \text { for } r=R
$$

where $r$ and $\hat{\mathbf{r}}$ are the length and the unit vector of the position vector $\mathbf{x}$, of which the origin is set to be the center of the strangelet. Condition (4) ensures the absence of the outgoing probability flux from the boundary. The analytic solutions to Eq. (3) under condition (4) were written [12,13] in the context of the MIT bag model; we adopt the solutions satisfying the normalization condition $\int_{V} d V\left(\Psi_{\xi \kappa a i}^{( \pm)}\right)^{\dagger} \Psi_{\xi \kappa a i}^{( \pm)}=1$. Such solutions read

$$
\Psi_{\xi \kappa a i}^{( \pm)}(\mathbf{x})=\left(\begin{array}{c}
g_{k \kappa i}^{( \pm)}(r) \\
-i f_{k \kappa i}^{( \pm)}(r) \boldsymbol{\sigma} \cdot \hat{\mathbf{r}}
\end{array}\right) \phi_{j m}^{l}(\Omega) \chi_{a}
$$

with

$$
g_{k \kappa i}^{( \pm)}(r)=A_{k \kappa i}^{( \pm)} j_{\kappa}(k r)+B_{k \kappa i}^{( \pm)} n_{\kappa}(k r)
$$




$$
f_{k \kappa i}^{( \pm)}(r)=\frac{k}{ \pm \omega_{k i}+m_{i}}\left[A_{k \kappa i}^{( \pm)} j_{\kappa-1}(k r)+B_{k \kappa i}^{( \pm)} n_{\kappa-1}(k r)\right] .
$$

Here $\chi_{a}$ is the color eigenstate, $j_{n}$ and $n_{n}$ are the $n$ th-order spherical Bessel functions,

$$
\phi_{j m}^{l=j+1 / 2}(\Omega)=\frac{1}{\sqrt{2(j+1)}}\left(\begin{array}{c}
\sqrt{j-m+1} Y_{j+1 / 2}^{m-1 / 2}(\Omega) \\
-\sqrt{j+m+1} Y_{j+1 / 2}^{m+1 / 2}(\Omega)
\end{array}\right) \quad \text { for } \kappa=j+1 / 2
$$

and

$$
\phi_{j m}^{l=j-1 / 2}(\Omega)=\frac{1}{\sqrt{2 j}}\left(\begin{array}{c}
\sqrt{j+m} Y_{j-1 / 2}^{m-1 / 2}(\Omega) \\
\sqrt{j-m} Y_{j-1 / 2}^{m+1 / 2}(\Omega)
\end{array}\right) \quad \text { for } \kappa=-(j+1 / 2)
$$

are the two-component spherical spinors, and

$$
A_{k \kappa i}^{( \pm)}= \begin{cases}\frac{k R}{\left|j_{\kappa}(k R)\right| R^{3 / 2}}\left[ \pm 2 \omega_{k i} R\left(\kappa \pm \omega_{k i} R\right)+m_{i} R\right]^{-1 / 2}, & \kappa=j+1 / 2, \\ 0, & \kappa=-(j+1 / 2),\end{cases}
$$

and

$$
B_{k \kappa i}^{( \pm)}= \begin{cases}0, & \kappa=j+1 / 2, \\ \frac{k R}{\left|j_{-\kappa-1}(k R)\right| R^{3 / 2}}\left[ \pm 2 \omega_{k i} R\left(\kappa \pm \omega_{k i} R\right)+m_{i} R\right]^{-1 / 2}, & \kappa=-(j+1 / 2),\end{cases}
$$

are the normalization factors. The energy eigenvalue $E= \pm \omega_{k i}$ may be determined from the resultant quantization condition 14

$$
j_{l=j \pm 1 / 2}(k R)=\mp \frac{k}{E+m_{i}} j_{l=j \mp 1 / 2}(k R) \quad \text { for } \kappa= \pm\left(j+\frac{1}{2}\right) .
$$

In the case of interest here, in which the baryon number $A$ is macroscopic, the solutions to Eq. (12) approximately read $k=N_{i} \pi / R$ with the principal quantum number $N_{i}=1,2,3, \ldots$. Here, the asymptotic form of $j_{l}(k R) \sim$ $(1 / k R) \cos [k R-(l+1) \pi / 2]$ for large $k R$ has been used, and only quantities of leading order in $k R$ have been retained. The $N_{i}$ th solution has a degree of degeneracy, $g_{N_{i}}=12\left(N_{i}+1\right)\left(2 N_{i}+1\right)$, stemming from the color, angular momentum $j=1 / 2,3 / 2, \ldots, 2 k R / \pi-3 / 2$, and parity $(-1)^{j \pm 1 / 2}$ [15]. We find from this $g_{N_{i}}$ that the Fermi wave number of $i$ quarks is $k_{F, i} \approx\left(\pi^{4} n_{i} / 6\right)^{1 / 3}$ with number density $n_{i}$, which is in turn related to $\mu_{i}$ as $\mu_{i}=\omega_{k_{F, i} i}$.

By building the resulting quark field $\psi_{a i}$ into Eq. (1) and by noting $\left\langle c_{\xi \kappa a i}^{\dagger} c_{\xi \kappa a i}\right\rangle=\theta\left(\mu_{i}-\omega_{k i}\right)$ and $\left\langle d_{\xi \kappa a i}^{\dagger} d_{\xi \kappa a i}\right\rangle=0$, calculations of $\Pi_{00}$ up to lowest order in $\mathbf{q}$ can be performed straightforwardly. The result, being of order $\mathbf{q}^{2}$ and expressed in terms of dipole transitions, can be formally divided as $\Pi_{00}(q)=\Pi_{00}^{(\mathrm{mat})}(q)+\Pi_{00}^{(\mathrm{vp})}(q)$. $\Pi_{00}^{(\mathrm{mat})}$ is the matter contribution given by

$$
\begin{aligned}
\Pi_{00}^{(\mathrm{mat})}(q)= & 2\left(\frac{g}{2}\right)^{2} \sum_{i} \sum_{\xi_{1} \xi_{2} \kappa_{1} \kappa_{2}}\left\{\theta\left(\mu_{i}-\omega_{k_{1} i}\right) \theta\left(\omega_{k_{2} i}-\mu_{i}\right)\right. \\
& \times\left(\frac{1}{\omega+i \eta+\omega_{k_{1} i}-\omega_{k_{2} i}}-\frac{1}{\omega+i \eta-\omega_{k_{1} i}+\omega_{k_{2} i}}\right) \mathcal{M}_{i \xi_{1} \xi_{2} \kappa_{1} \kappa_{2}}^{(q q)}(\mathbf{q})+\theta\left(\mu_{i}-\omega_{k_{1} i}\right) \\
& \left.\times\left(\frac{1}{\omega+i \eta+\omega_{k_{1} i}+\omega_{k_{2} i}}-\frac{1}{\omega+i \eta-\omega_{k_{1} i}-\omega_{k_{2} i}}\right) \mathcal{M}_{i \xi_{1} \xi_{2} \kappa_{1} \kappa_{2}}^{(\bar{q} q)}(\mathbf{q})\right\},
\end{aligned}
$$

with

$$
\begin{aligned}
\mathcal{M}_{i \xi_{1} \xi_{2} \kappa_{1} \kappa_{2}}^{(q q)}= & -\frac{1}{2 V} \int_{V} d \mathbf{x} \int_{V} d \mathbf{y}[\mathbf{q} \cdot(\mathbf{x}-\mathbf{y})]^{2} \Psi_{\xi_{1} \kappa_{1} a_{1} i}^{(+) \dagger}(\mathbf{x}) \Psi_{\xi_{2} \kappa_{2} a_{2} i}^{(+)}(\mathbf{x}) \Psi_{\xi_{2} \kappa_{2} a_{2} i}^{(+) \dagger}(\mathbf{y}) \Psi_{\xi_{1} \kappa_{1} a_{1} i}^{(+)}(\mathbf{y}) \\
= & \frac{\mathbf{q}^{2}}{4 V}\left|\left\langle k_{1}, j_{1}, \kappa_{1}, i,+|r| k_{2}, j_{2}, \kappa_{2}, i,+\right\rangle\right|^{2}\left[\frac{\left(j_{1}-m_{1}+1\right)\left(j_{1}+m_{1}+1\right)}{\left(j_{1}+1\right)^{2}}\right. \\
& \times \delta_{j_{1}+1, j_{2}} \delta_{m_{1}, m_{2}} \theta\left(\kappa_{1} \kappa_{2}\right)+\frac{\left(j_{1}-m_{1}\right)\left(j_{1}+m_{1}\right)}{j_{1}^{2}} \delta_{j_{1}-1, j_{2}} \delta_{m_{1}, m_{2}} \theta\left(\kappa_{1} \kappa_{2}\right) \\
& \left.+\frac{m_{1}^{2}}{j_{1}^{2}\left(j_{1}+1\right)^{2}} \delta_{j_{1}, j_{2}} \delta_{m_{1}, m_{2}} \theta\left(-\kappa_{1} \kappa_{2}\right)\right]
\end{aligned}
$$




$$
\begin{aligned}
\mathcal{M}_{i \xi_{1} \xi_{2} \kappa_{1} \kappa_{2}}^{(\bar{q} q)}= & -\frac{1}{2 V} \int_{V} d \mathbf{x} \int_{V} d \mathbf{y}[\mathbf{q} \cdot(\mathbf{x}-\mathbf{y})]^{2} \Psi_{\xi_{1} \kappa_{1} a_{1} i}^{(+) \dagger}(\mathbf{x}) \Psi_{\xi_{2} \kappa_{2} a_{2} i}^{(-)}(\mathbf{x}) \Psi_{\xi_{2} \kappa_{2} a_{2} i}^{(-) \dagger}(\mathbf{y}) \Psi_{\xi_{1} \kappa_{1} a_{1} i}^{(+)}(\mathbf{y}) \\
= & \frac{\mathbf{q}^{2}}{4 V}\left|\left\langle k_{1}, j_{1}, \kappa_{1}, i,+|r| k_{2}, j_{2}, \kappa_{2}, i,-\right\rangle\right|^{2}\left[\frac{\left(j_{1}-m_{1}+1\right)\left(j_{1}+m_{1}+1\right)}{\left(j_{1}+1\right)^{2}}\right. \\
& \times \delta_{j_{1}+1, j_{2}} \delta_{m_{1}, m_{2}} \theta\left(\kappa_{1} \kappa_{2}\right)+\frac{\left(j_{1}-m_{1}\right)\left(j_{1}+m_{1}\right)}{j_{1}^{2}} \delta_{j_{1}-1, j_{2}} \delta_{m_{1}, m_{2}} \theta\left(\kappa_{1} \kappa_{2}\right) \\
& \left.+\frac{m_{1}^{2}}{j_{1}^{2}\left(j_{1}+1\right)^{2}} \delta_{j_{1}, j_{2}} \delta_{m_{1}, m_{2}} \theta\left(-\kappa_{1} \kappa_{2}\right)\right]
\end{aligned}
$$

and $\Pi_{00}^{(\mathrm{vp})}$ is the vacuum contribution given by

$$
\begin{aligned}
\Pi_{00}^{(\mathrm{vp})}(q)= & -2\left(\frac{g}{2}\right)^{2} \sum_{i} \sum_{\xi_{1}}\left(\frac { 1 } { \xi _ { 2 } \kappa _ { 1 } \kappa _ { 2 } } \left(\frac{1}{\omega+i \eta+\omega_{k_{1} i}+\omega_{k_{2} i}}-\frac{1}{\omega+i \eta-\omega_{k_{1} i}-\omega_{k_{2} i}}\right.\right. \\
& \left.-\frac{2}{\omega_{k_{1} i}+\omega_{k_{2} i}}\right) \mathcal{M}_{i \xi_{1} \xi_{2} \kappa_{1} \kappa_{2}}^{(\bar{q} q)}(\mathbf{q}) .
\end{aligned}
$$

Here $\eta$ is a positive infinitesimal, and $\left\langle k_{1}, j_{1}, \kappa_{1}, i,+|r| k_{2}, j_{2}, \kappa_{2}, i, \pm\right\rangle$, in which the sign $+(-)$ specifies the quark (antiquark) state, denote the radial components of the matrix elements. These components vanish when $k_{1}=k_{2}$, or else they are given by

$$
\begin{aligned}
& \left|\left\langle k_{1}, j_{1}, \kappa_{1}= \pm\left(j_{1}+1 / 2\right), i,+|r| k_{2}, j_{2}=j_{1}+1, \kappa_{2}= \pm\left(j_{1}+3 / 2\right), i,+\right\rangle\right|^{2} \\
& =\left[\frac{2 k_{1} k_{2}}{\left(k_{1}^{2}-k_{2}^{2}\right)^{2} R}\right]^{2} \frac{\left[\left(\omega_{k_{1} i}+\omega_{k_{2} i}\right)^{2} R^{2}+\left(\kappa_{1}+\kappa_{2}\right)\left(\omega_{k_{1} i}+\omega_{k_{2} i}\right) R\right]^{2}}{\left[2 \omega_{k_{1} i} R\left(\kappa_{1}+\omega_{k_{1} i} R\right)+m_{i} R\right]\left[2 \omega_{k_{2} i} R\left(\kappa_{2}+\omega_{k_{2} i} R\right)+m_{i} R\right]}, \\
& \left|\left\langle k_{1}, j_{1}, \kappa_{1}= \pm\left(j_{1}+1 / 2\right), i,+|r| k_{2}, j_{2}=j_{1}-1, \kappa_{2}= \pm\left(j_{1}-1 / 2\right), i,+\right\rangle\right|^{2} \\
& =\left[\frac{2 k_{1} k_{2}}{\left(k_{1}^{2}-k_{2}^{2}\right)^{2} R}\right]^{2} \frac{\left[\left(\omega_{k_{1} i}+\omega_{k_{2} i}\right)^{2} R^{2}+\left(\kappa_{1}+\kappa_{2}\right)\left(\omega_{k_{1} i}+\omega_{k_{2} i}\right) R\right]^{2}}{\left[2 \omega_{k_{1} i} R\left(\kappa_{1}+\omega_{k_{1} i} R\right)+m_{i} R\right]\left[2 \omega_{k_{2} i} R\left(\kappa_{2}+\omega_{k_{2} i} R\right)+m_{i} R\right]}, \\
& \left|\left\langle k_{1}, j_{1}, \kappa_{1}= \pm\left(j_{1}+1 / 2\right), i,+|r| k_{2}, j_{2}=j_{1}, \kappa_{2}=\mp\left(j_{1}+1 / 2\right), i,+\right\rangle\right|^{2} \\
& =\left(\frac{2 k_{1} k_{2} R}{k_{1}^{2}-k_{2}^{2}}\right)^{2} \frac{\left[\left(\omega_{k_{1} i}-\omega_{k_{2} i}\right) R+\frac{2 m_{i}}{\omega_{k_{1} i}-\omega_{k_{2} i}}+\left(\kappa_{1}-\kappa_{2}\right)\right]^{2}}{\left[2 \omega_{k_{1} i} R\left(\kappa_{1}+\omega_{k_{1} i} R\right)+m_{i} R\right]\left[2 \omega_{k_{2} i} R\left(\kappa_{2}+\omega_{k_{2} i} R\right)+m_{i} R\right]}, \\
& \left|\left\langle k_{1}, j_{1}, \kappa_{1}= \pm\left(j_{1}+1 / 2\right), i,+|r| k_{2}, j_{2}=j_{1}+1, \kappa_{2}= \pm\left(j_{1}+3 / 2\right), i,-\right\rangle\right|^{2} \\
& =\left[\frac{2 k_{1} k_{2}}{\left(k_{1}^{2}-k_{2}^{2}\right)^{2} R}\right]^{2} \frac{\left[\left(\omega_{k_{1} i}-\omega_{k_{2} i}\right)^{2} R^{2}+\left(\kappa_{1}+\kappa_{2}\right)\left(\omega_{k_{1} i}-\omega_{k_{2} i}\right) R\right]^{2}}{\left[2 \omega_{k_{1} i} R\left(\kappa_{1}+\omega_{k_{1} i} R\right)+m_{i} R\right]\left[2 \omega_{k_{2} i} R\left(-\kappa_{2}+\omega_{k_{2} i} R\right)+m_{i} R\right]}, \\
& \left|\left\langle k_{1}, j_{1}, \kappa_{1}= \pm\left(j_{1}+1 / 2\right), i,+|r| k_{2}, j_{2}=j_{1}-1, \kappa_{2}= \pm\left(j_{1}-1 / 2\right), i,-\right\rangle\right|^{2} \\
& =\left[\frac{2 k_{1} k_{2}}{\left(k_{1}^{2}-k_{2}^{2}\right)^{2} R}\right]^{2} \frac{\left[\left(\omega_{k_{1} i}-\omega_{k_{2} i}\right)^{2} R^{2}+\left(\kappa_{1}+\kappa_{2}\right)\left(\omega_{k_{1} i}-\omega_{k_{2} i}\right) R\right]^{2}}{\left[2 \omega_{k_{1} i} R\left(\kappa_{1}+\omega_{k_{1} i} R\right)+m_{i} R\right]\left[2 \omega_{k_{2} i} R\left(-\kappa_{2}+\omega_{k_{2} i} R\right)+m_{i} R\right]}, \\
& \left|\left\langle k_{1}, j_{1}, \kappa_{1}= \pm\left(j_{1}+1 / 2\right), i,+|r| k_{2}, j_{2}=j_{1}, \kappa_{2}=\mp\left(j_{1}+1 / 2\right), i,-\right\rangle\right|^{2} \\
& =\left(\frac{2 k_{1} k_{2} R}{k_{1}^{2}-k_{2}^{2}}\right)^{2} \frac{\left[\left(\omega_{k_{1} i}+\omega_{k_{2} i}\right) R+\frac{2 m_{i}}{\omega_{k_{1} i}+\omega_{k_{2} i}}+\left(\kappa_{1}-\kappa_{2}\right)\right]^{2}}{\left[2 \omega_{k_{1} i} R\left(\kappa_{1}+\omega_{k_{1} i} R\right)+m_{i} R\right]\left[2 \omega_{k_{2} i} R\left(-\kappa_{2}+\omega_{k_{2} i} R\right)+m_{i} R\right]} .
\end{aligned}
$$

It is convenient to further divide the matter part $\Pi_{00}^{(\mathrm{mat})}$ as $\Pi_{00}^{(\mathrm{mat})}=\Pi_{00}^{(0)}+\Pi_{00}^{(\mathrm{so})}+\Pi_{00}^{(\bar{q} q)}$, where $\Pi_{00}^{(0)}$, $\Pi_{00}^{(\mathrm{so})}$, and $\Pi_{00}^{(\bar{q} q)}$ are composed of the matrix elements written by Eqs. (17) and (18), Eq. (19), and Eqs. (20)-(22), respectively. 
We thus find that $\Pi_{00}^{(0)}\left(\Pi_{00}^{(\mathrm{so})}\right)$ arises from excitations of quark particle-hole pairs, accompanied by the change in the angular momentum of the scattered quark $\Delta j \equiv j_{2}-j_{1}= \pm 1(\Delta j=0) ; \Pi_{00}^{(\bar{q} q)}\left(\Pi_{00}^{(\mathrm{vp})}\right)$ arises from excitations of quark-antiquark pairs via scattering of the quark in the filled Fermi (Dirac) sea, accompanied by $\Delta j=0, \pm 1$, onto the vacant Dirac (Fermi) sea. The quantities $\Pi_{00}^{(\mathrm{so})}, \Pi_{00}^{(\bar{q} q)}$, and $\Pi_{00}^{(\mathrm{vp})}$ are associated with effects of relativity: the spin-orbit interactions, fluctuations of the Fermi sea involving quark-antiquark pairs, and the second-order vacuum polarization, respectively.

\section{DISPERSION RELATION}

The dispersion relation of the longitudinal color plasma oscillation has been obtained from the one-loop polarization $\Pi_{00}$ given by Eqs. (13) and (16). By noting the complete analogy with the case of a metal nanoparticle in a dielectric medium [10], such a relation can be written as

$$
\left[\frac{1}{3} \epsilon_{0}-\frac{\operatorname{Re} \Pi_{00}(q)}{\mathbf{q}^{2}}\right]+\frac{2}{3} \epsilon_{\mathrm{vac}}=0
$$

where $\epsilon_{0}$ is the color dielectric constant of the strangelet, having the color carrier part subtracted out, and $\epsilon_{\mathrm{vac}}$ is that of the surrounding vacuum. We normally set $\epsilon_{0}=1$ and $\epsilon_{\mathrm{vac}}=0$. Up to leading order in $k_{F, i} R$, the summation over $\xi_{1}, \xi_{2}, \kappa_{1}$, and $\kappa_{2}$ in Eqs. (13) and (16) may be performed by, if available, replacing $\sum_{j} \rightarrow \int_{0}^{2 k R / \pi} d j$ and $\sum_{k} \rightarrow(R / \pi) \int_{0}^{k_{F, i}} d k$. The main contribution to $\operatorname{Re} \Pi_{00}$ is $\operatorname{Re} \Pi_{00}^{(0)}=\mathbf{q}^{2} \omega_{p}^{2} / 3 \omega^{2}$, where $\omega_{p}=\sqrt{\sum_{i} g^{2} n_{i} / 6 \mu_{i}}$ is the color plasma frequency of bulk quark matter [7,8]. $\left|\operatorname{Re} \Pi_{00}^{(\mathrm{so})}\right| / \mathbf{q}^{2}\left(\left|\operatorname{Re} \Pi_{00}^{(\bar{q} q)}+\operatorname{Re} \Pi_{00}^{(\mathrm{vp})}\right| / \mathbf{q}^{2}\right)$ is suppressed by one power $1 / k_{F, i} R\left(\alpha_{s}\right)$ with respect to $\left|\operatorname{Re} \Pi_{00}^{(0)}\right| / \mathbf{q}^{2}$ (unity), where $\alpha_{s}=g^{2} / 4 \pi$ is the QCD fine structure constant. We thus obtain the mode frequency $\omega \approx \omega_{p}$. Its agreement with the bulk value stems from absence of the surface color charge due to $\epsilon_{\mathrm{vac}}=0$. The values of $\omega_{p}$ calculated as a function of $\eta_{s}=m_{s} / \mu$ for $\alpha_{s}=0.4$ have been exhibited in Fig. 1 . We have found that $\omega_{p}$ depends on $m_{s}$ only weakly.

\section{DAMPING RATE}

Within the framework of the RPA, the damping of the longitudinal color oscillation mode is determined by its resonant energy transfer into individual excitations of particle-hole pairs of the quarks whose eigenenergies are made discrete by the boundary. The corresponding damping rate may be derived from the polarization $\Pi_{00}$ obtained above as $[8]$

$$
\Gamma_{\mathrm{RPA}}=-\left.\frac{3 \omega_{p}}{2 \mathbf{q}^{2}} \operatorname{Im} \Pi_{00}(q)\right|_{\omega=\omega_{p}}
$$

Here $\Gamma_{\mathrm{RPA}} \ll \omega_{p}$ is assumed, and the imaginary part of $\Pi_{00}(q)$ is expressed up to leading order in $k_{F, i} R$ and $\mathbf{q}$ as

$$
\operatorname{Im} \Pi_{00}(q)=-\frac{4 g^{2}}{\pi^{4} \mu R} \mathbf{q}^{2} \sum_{i} \frac{h\left(\nu, \eta_{i}\right)}{\nu^{3}}
$$

with $\nu=\omega / \mu, \eta_{i}=m_{i} / \mu$, and

$$
\begin{aligned}
h\left(\nu, \eta_{i}\right)= & \frac{1}{\nu} \int_{\max \left(1-\nu, \eta_{i}\right)}^{1} d z \frac{\sqrt{z^{2}-\eta_{i}^{2}} \sqrt{(z+\nu)^{2}-\eta_{i}^{2}}}{(2 z+\nu)^{2}} \\
& \times\left[\left(z^{2}-\eta_{i}^{2}\right)-\frac{\pi^{2} \nu\left(8 z^{3}+8 \nu z^{2}+4 \nu^{2} z+\nu^{3}\right)}{64 z(2 z+\nu)} \ln \left|1-\left(\frac{2}{\pi}\right)^{2} \frac{z^{2}-\eta_{i}^{2}}{z^{2}}\right|\right. \\
& +\frac{\pi^{2} \nu\left(8 z^{3}+16 \nu z^{2}+12 \nu^{2} z+3 \nu^{3}\right)}{64(z+\nu)(2 z+\nu)} \ln \left|1-\left(\frac{2}{\pi}\right)^{2} \frac{z^{2}-\eta_{i}^{2}}{(z+\nu)^{2}}\right| \\
& \left.+\frac{\pi^{2} \nu^{4}}{32 z(z+\nu)}\left(1+\ln \left|1+\frac{2}{\pi} \sqrt{z^{2}-\eta_{i}^{2}} \mu R\right|\right)\right] .
\end{aligned}
$$


In Fig. 2, we have shown $\Gamma_{\mathrm{RPA}} / \omega_{p}$ as a function of $R$ for various values of $\eta_{s}$. For $\omega \leq \mu+m_{i}$, which is satisfied at $\omega=\omega_{p}, \operatorname{Im} \Pi_{00}^{(\mathrm{vp})}$ cancels out $\operatorname{Im} \Pi_{00}^{(\bar{q} q)}$. On the other hand, the contributions of $\operatorname{Im} \Pi_{00}^{(0)}$ and $\operatorname{Im} \Pi_{00}^{(\mathrm{so})}$, the latter being suppressed by one power $\alpha_{s}$ with respect to the former, lead to the damping of which the rate behaves as $\sim c / R$ [16]. Such contributions may be regarded as Landau damping, i.e., a decay of the collective mode by exciting a single real particle-hole pair. In this case, the surface of the strangelet, which acts as a boundary condition determining the discrete quark levels rather than as a scatterer, renders $\Gamma_{\mathrm{RPA}}$ nonzero quantum mechanically and independent of $\alpha_{s}$ in the weak-coupling limit. As can be observed from Fig. 2, $\Gamma_{\mathrm{RPA}}$ has only a slight dependence on $m_{s}$. In the nonrelativistic limit, only the part $\operatorname{Im} \Pi_{00}^{(0)}$ remains up to $O\left(1 / k_{F, i} R\right)$, still yielding $\Gamma_{\mathrm{RPA}} \propto R^{-1}$. We remark in passing that for a strangelet with radius $R \lesssim 3 \mathrm{fm}$, which has a baryon number of less than $\sim 30$, higher-order terms in $\left(k_{F, i} R\right)^{-1}$ as ignored here may well have consequence to the frequency and damping rate of the color plasmons.

We next consider the damping of the longitudinal color plasmons due to collisions 17 between quarks in the weakcoupling regime. These collisions, by color exchange, disrupt the self-consistent fields responsible for the plasmons and add $\Gamma_{\text {col }}$ to the RPA result $\Gamma_{\mathrm{RPA}}$. If one attempts to calculate $\Gamma_{\text {col }}$ in perturbation theory, one meets an infrared divergence that arises from the color magnetic interactions mediated by transverse gluons. At least for finite temperature [18], such a divergence cannot be removed even by dynamical screening [19] of the magnetic interactions [20]. This indicates that calculations of $\Gamma_{\text {col }}$ up to leading order in $\alpha_{s}$ require nonperturbative features of the relativistic quark-gluon plasma which may possibly develop a magnetic mass $m_{\text {mag }}$ and thereby provide an effective cutoff [21]. For a thermal, massless plasma at zero baryon density, where $m_{\text {mag }} \sim \alpha_{s} T$, one thus obtains $\Gamma_{\text {col }} \sim \alpha_{s} \ln \left(1 / \alpha_{s}\right) T$ from kinetic theory [17]. This rate arises from the collision integral associated with one-gluon exchange in the bulk plasma; finite-size corrections of $O\left(1 / k_{F, i} R\right)$ to such a bulk result for $\Gamma_{\text {col }}$ should be of higher order in $\alpha_{s}$ than $\Gamma_{\mathrm{RPA}}$, which remains in the absence of the collision integral. At the nonzero strange quark mass, baryon density, and temperature of interest here, however, the values of $m_{\text {mag }}$ and hence of $\Gamma_{\text {col }}$ are essentially unknown. Therefore, all we can mention is that such a collisional damping or the Landau damping due to individual excitations of quark particle-hole pairs dominates the total damping rate $\Gamma_{\text {tot }}=\Gamma_{\mathrm{RPA}}+\Gamma_{\text {col }}$, according to whether the mean free path of the quarks $\sim \Gamma_{\text {col }}^{-1}$ is small or large compared with $R$. Here, it is instructive to note that no damping of the plasmons due to gluon radiation occurs in the absence of the surface color charge.

\section{CONCLUDING REMARKS}

We have calculated the dispersion relation and damping rate of the longitudinal color plasma oscillations in a strangelet in the regimes of weak coupling, low temperature, and long wavelength. For $k_{F, i} R \gg 1$, we have found that the frequency of such oscillations is almost as large as that of bulk color plasmons and that the Landau damping yields a lifetime proportional to $R$. However, the present analysis is accompanied by several uncertainties. We have confined ourselves to the case of low $|\mathbf{q}|$ and $T$. With increasing $|\mathbf{q}|$, multipole plasmon excitations should develop. At finite temperature, furthermore, gluonic excitations in the intermediate state contribute to the proper polarization $\Pi_{00}$; within the one-loop approximation, such a contribution is simply added to the quark-loop contribution without interference [8]. A study allowing for those excitations has yet to be done. Shell effects in strangelets also remain to be taken into account; such effects are predicted to manifest themselves remarkably for $A \lesssim 100[6,22]$. It is expected from the analogy with the case of collective excitations in normal nuclei that whether strangelets are in the vicinity of closed shells or not plays a role in the properties of the color plasma oscillations. Third, it is to be noted that the present analysis is restricted to the weak-coupling regime $\left(\alpha_{s} \ll 1\right)$. In the strongly interacting nonperturbative regime $\left(\alpha_{s} \sim 1\right)$, more relevant to a real system, the damping rate of the color plasmons could be greatly modified. These nonperturbative effects should have primary significance to the collisional damping.

To conclude, we briefly address what type of traces a strangelet, if formed in heavy-ion collision experiments 23] performed at a beam energy of order $200 \mathrm{GeV}$ per nucleon on a fixed target, could leave via excitations of the longitudinal color plasmons. Immediately after central nucleus-nucleus collisions at such energies, it is expected that a quark-gluon plasma would be produced in the resulting matter of sufficiently high energy density. Then, the quarkgluon plasma might develop into strangelets via pre-freeze-out evaporation of pions, $K^{+}$, and $K^{0}$ which carry away entropy and antistrangeness from the system. This strangeness distillation mechanism, suggested by Greiner et al. [24], would work as long as strangelets are stable against strong decay. Note that the freezeout temperature $\sim 100-$ $200 \mathrm{MeV} 25$ is comparable with the frequency $\omega_{p}$ for realistic values of $\alpha_{s}$ in the range $\sim 0.4-1$. Longitudinal color plasma oscillations would thus be thermally excited in strangelets until the temperature fully drops. On a time scale

of order $\Gamma_{\text {tot }}^{-1}$, the excited color plasmons would decay into quark particle-hole pairs, leading to emission of photons and lepton pairs via electromagnetic interactions. These electromagnetic signals, having energy of order or less than $\sim \omega_{p}$, might be observed in the highly energetic heavy-ion collisions at CERN's SPS experiments [23]. In order to support the picture mentioned above, however, estimates allowing for relaxation processes occurring in such a cooled and 
decompressed system, for other mechanisms producing photons and lepton pairs, and for the uncertainties discussed in the preceding paragraph, are required. Elucidation of this problem is beyond the scope of this paper.

\section{ACKNOWLEDGMENTS}

The author thanks G. Baym for useful discussion. He acknowledges the hospitality of Aspen Center for Physics, where this work was initiated. This work was supported in part by a Grant-in-Aid for Scientific Research provided by the Ministry of Education, Science, and Culture of Japan through Grants Nos. 07CE2002 and 199803687.

[1] See, e.g., G. Baym, in Elementary Processes in Dense Plasmas, edited by S. Ichimaru and S. Ogata (Addison-Wesley, Reading, MA, 1995), p. 3; A. V. Smilga, Phys. Rep. 291, 1 (1997).

[2] E. Witten, Phys. Rev. D 30, 272 (1984).

[3] M. Brügger, K. Lützenkirchen, S. Polikanov, G. Herrmann, M. Overbeck, N. Trautmann, A. Breskin, R. Chechik, Z. Fraenkel, and U. Smilansky, Nature (London) 337, 434 (1989).

[4] T. A. Armstrong et al., Phys. Rev. Lett. 79, 3612 (1997).

[5] M. C. Perillo Isaac et al., Phys. Rev. Lett. 81, 2416 (1998).

[6] E. Farhi and R. L. Jaffe, Phys. Rev. D 30, 2379 (1984).

[7] G. Baym and S. A. Chin, Nucl. Phys. A262, 527 (1976).

[8] O. K. Kalashnikov, Fortschr. Phys. 32, 525 (1984).

[9] A. Kawabata and R. Kubo, J. Phys. Soc. Jpn. 21, 1765 (1966).

[10] U. Kreibig and M. Vollmer, Optical Properties of Metal Clusters (Springer-Verlag, Berlin, 1995).

[11] B. Jancovici, Nuovo Cimento 25, 428 (1962).

[12] T. DeGrand, R. L. Jaffe, K. Johnson, and J. Kiskis, Phys. Rev. D 12, 2060 (1975).

[13] D. Vasak, W. Greiner, and L. Neise, Phys. Rev. C 34, 1307 (1986).

[14] I. Mardor and B. Svetitsky, Phys. Rev. D 44, 878 (1991).

[15] The rigorous energy spectrum has nondegenerate levels for each $\left(N_{i}, j, \kappa\right)$. For the large $A$ of interest here, however, it is sufficient to note that the eigenstates having $N_{i}$, of which the number is identical with $g_{N_{i}}$, lie in the range $N_{i-1} \pi / R<$ $k<N_{i+1} \pi / R$.

[16] As can be seen from Eq. (26), the rate $\Gamma_{\mathrm{RPA}}$ includes a term of order $R^{-1} \ln k_{F, i} R$ induced by the spin-orbit interactions. This term is negligibly small compared with the term of order $R^{-1}$ except for anomalously large strangelets.

[17] A. V. Selikhov and M. Gyulassy, Phys. Lett. B 316, 373 (1993); H. Heiselberg, Phys. Rev. Lett. 72, 3013 (1994).

[18] At nonzero temperature, not only quarks but also gluons and antiquarks act as real color carriers. These gluons and antiquarks participate in the color plasmon mode, while their collisions with other colored particles yield an additional contribution to $\Gamma_{\text {col }}$. As long as $T \ll \mu$, however, such effects make little difference.

[19] H. A. Weldon, Phys. Rev. D 26, 1394 (1982).

[20] Using the field-theoretical approach involving the resummation of the so-called hard thermal loops into effective propagators and vertices, E. Braaten and R. D. Pisarski [Phys. Rev. Lett. 64, 1338 (1990); Phys. Rev. D 42, 2156 (1990); Nucl. Phys. B337, 569 (1990)] concluded that to leading order in $\alpha_{s}$, the collisional damping rate of the color plasmon at $\mu=m_{s}=|\mathbf{q}|=0$ is infrared finite $\left(\sim \alpha_{s} T\right)$ and gauge independent. However, it has been suggested by analyzing the behavior of the effective gluon propagator in the vicinity of the mass shell (Smilga 1]) that such a rate of order $\alpha_{s} T$ is still gauge dependent and thus is not a physical quantity.

[21] As an additional mechanism for possibly regularizing the infrared divergence, see J. P. Blaizot and E. Iancu, Phys. Rev. Lett. 76, 3080 (1996).

[22] E. P. Gilson and R. L. Jaffe, Phys. Rev. Lett. 71, 332 (1993).

[23] Reviewed in Proceedings of Quark Matter '97 [Nucl. Phys. A638, 1c (1998)].

[24] C. Greiner, P. Koch, and H. Stöcker, Phys. Rev. Lett. 58, 1825 (1987); C. Greiner, D. H. Rischke, H. Stöcker, and P. Koch, Phys. Rev. D 38, 2797 (1988); C. Greiner and H. Stöcker, ibid. 44, 3517 (1991).

[25] U. Heinz, J. Phys. G 25, 263 (1999). 


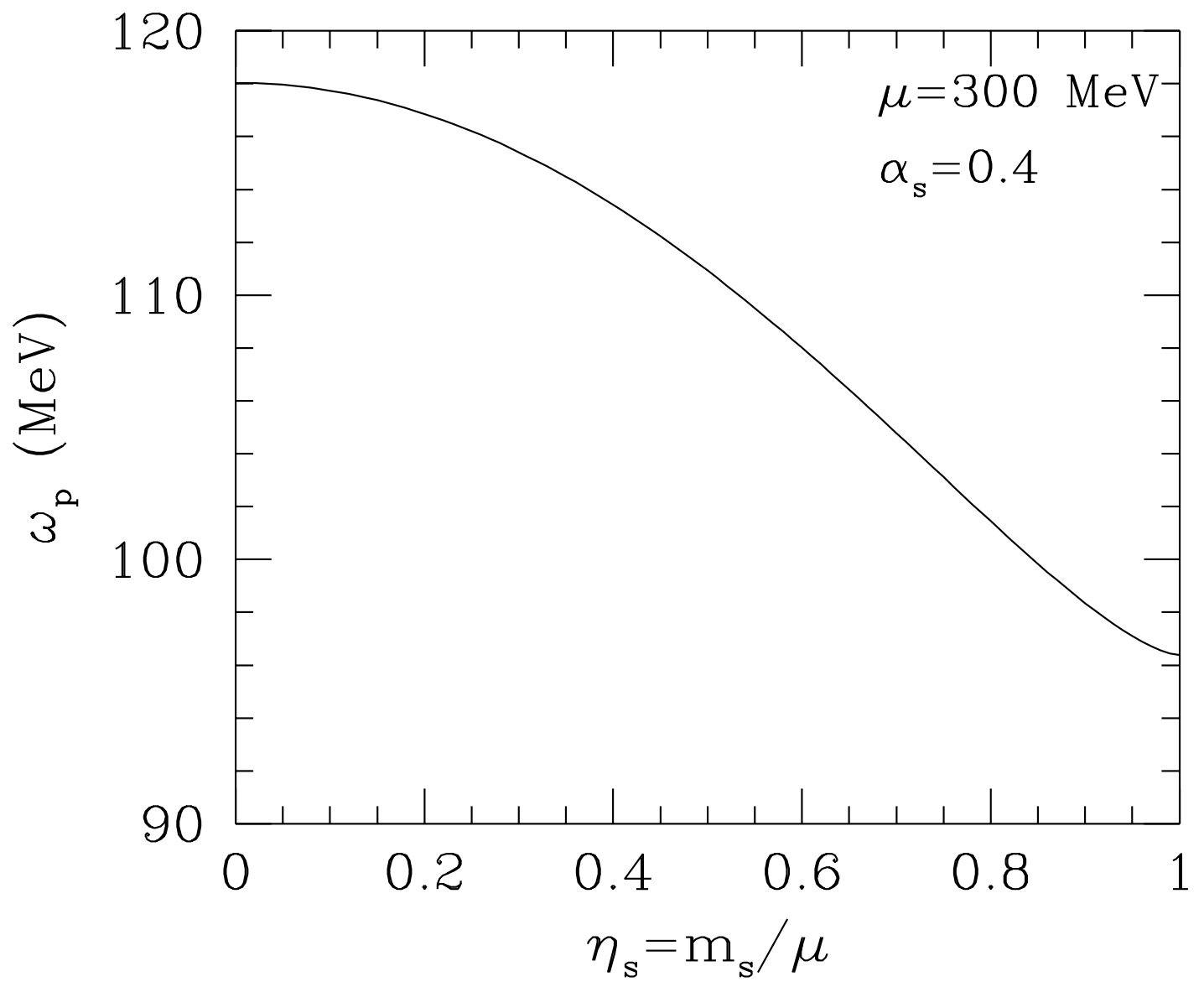

FIG. 1. Color plasma frequency $\omega_{p}$ as a function of $\eta_{s}$, calculated for $\mu=300 \mathrm{MeV}$ and $\alpha_{s}=0.4$. 


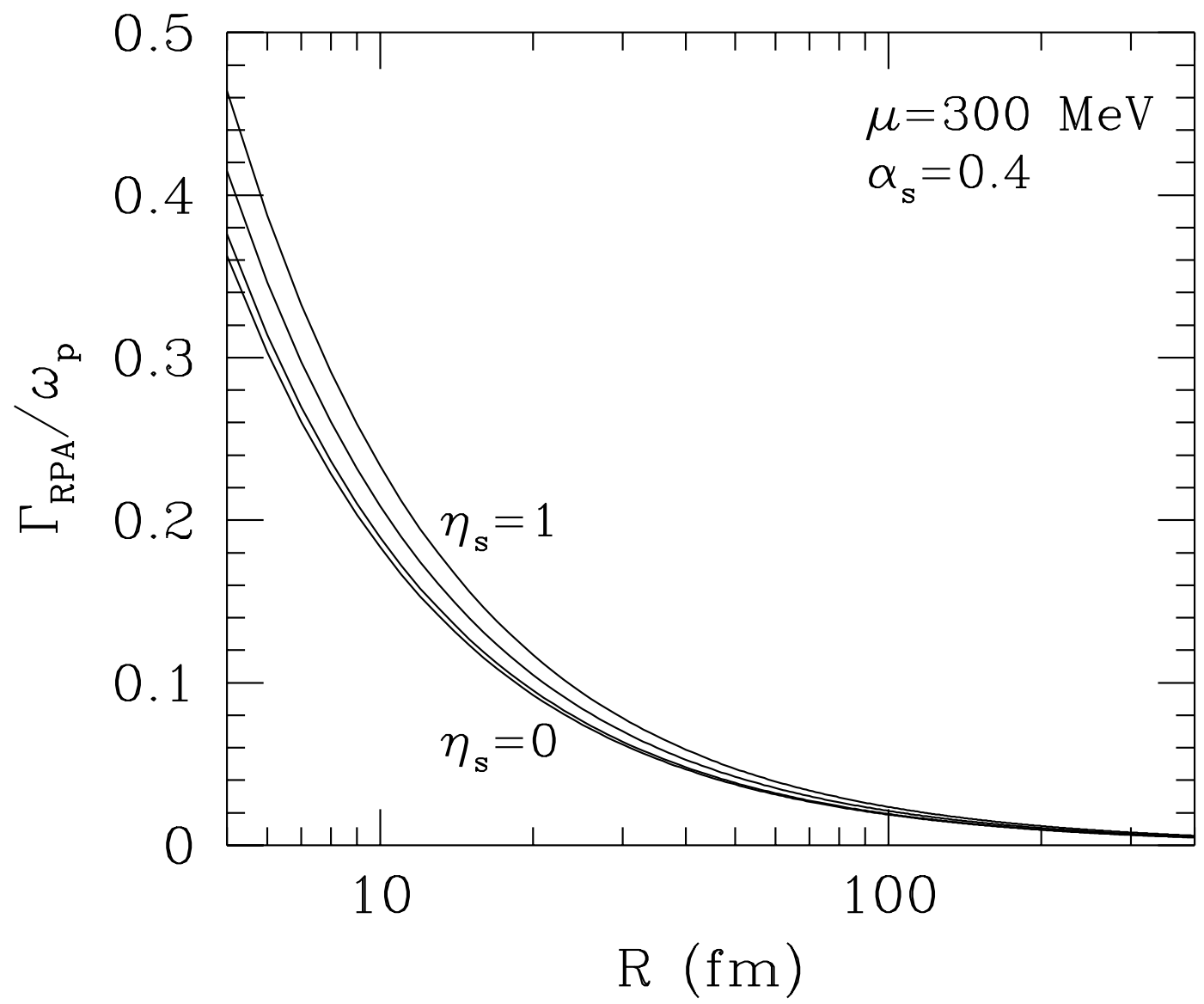

FIG. 2. Damping rates $\Gamma_{\mathrm{RPA}}$ divided by $\omega_{p}$ as a function of the strangelet radius $R$, which have been evaluated for $\mu=300$ $\mathrm{MeV}$ and $\alpha_{s}=0.4$. The lines from bottom to top are the results obtained for $\eta_{s}=0,2 / 3,5 / 6,1$. 